\title{
Decay of Temperature Fluctuations in MHD Turbulence before the Final Period in a Rotating System
}

\author{
M. Abul Kalam Azad ${ }^{a *}$ and M. S. Alam Sarker \\ Department of Applied Mathematics, University of Rajshahi Rajshahi-6205, Bangladesh
}

\begin{abstract}
Using Deissler's approach the Decay of temperature fluctuations in MHD turbulence before the final period in a rotating system is studied and have considered correlations between fluctuating quantities at two and three point. In this case two and three-point correlation equations in a rotating system is obtained and the set of equations is made to determinate by neglecting the quadruple correlations in comparison to the second and third order correlations. The correlation equations are converted to special form by taking their Fourier-transforms. Finally integrating the energy spectrum over all wave numbers the energy decay law of temperature fluctuations in MHD turbulence before the final period in a rotating system is obtained.
\end{abstract}

Key words : Deissler's method, MHD turbulence, Rotating system, Temperature fluctuation.

\section{Introduction}

Deissler (1958 and 1960) developed a theory for homogeneous turbulence, which was valid for times before the final period. Using Deissler's theory Loeffler and Deissler (1961) studied the decay of temperature fluctuations in homogeneous turbulence before the final period. Following Deissler's approach Sarker and Islam (2001) also studied the decay of temperature fluctuations in homogeneous turbulence before the final period for the case of multi-point and multi-time. Sarker and L. Rahman (1998) studied the decay of temperature fluctuations in MHD turbulence before the final period. Islam and Sarker (2001) studied the first order reactant in MHD turbulence before the final period of decay for the case of multi-point and multi-time. Kumar and Patel (1975) also studied on fist-order reactant in homogeneous turbulence before the final period of decay for the case of multipoint and multi-time. Sarker and Islam (2001) studied the decay of MHD turbulence before the final period for the case of multi-point and multi-time. Further work along this same line for the case of multi-point and single time had been done by Sarker and Kishore (1991).

In their approach they considered two and three-point correlations after neglecting higher order correlation terms compared to the second-and third-order correlation terms.

Kishore and Dixit (1979), Kishore and Singh (1984) discussed the effect of coriolis force on acceleration covariance in ordinary and MHD turbulence. Shimomura and Yoshizawa (1986), Shimomura (1986 and 1989) also

\footnotetext{
* Corresponding author: E-mail: azad_amath64@yahoo.com
}

discussed the statistical analysis of turbulent viscosity, turbulent scalar flux and turbulent shear flows respectively in a rotating system by two-scale direct interaction approach. Sarker and Islam (2001) studied the decay of dusty fluid turbulence before the final period in rotating system. Azad, Sarker and Mondol (2006) studied the decay of temperature fluctuations in dusty fluid MHD turbulence before the final period in a rotating system and also Azad and Sarker (2003) decay of MHD turbulence before the final period for the case of multi-point and multi-time in presence of dust particle has more recently been done by Azad and Sarker (2003).

By analyzing the above theories we have studied the decay of temperature fluctuations in MHD turbulence before the final period in a rotating system using two-and three-point correlation equations neglecting fourth order correlation terms compared to the second-and third-order correlation terms. Finally, the energy decay law of temperature fluctuations in MHD turbulence before the final period in a rotating system is obtained.

\section{Basic Equations}

The equation of motion and continuity for viscous, incompressible MHD turbulent flow in a rotating system are given by

$$
\frac{\partial u_{i}}{\partial t}+\frac{\partial}{\partial x_{k}}\left(u_{i} u_{k}-h_{i} h_{k}\right)=\frac{\partial w}{\partial x_{i}}+v \frac{\partial^{2} u_{i}}{\partial x_{k} \partial x_{k}}-2 \epsilon_{m k i} \Omega_{h} u_{i},--(1)
$$


$\frac{\partial h_{i}}{\partial t}+\frac{\partial}{\partial x_{k}}\left(h_{i} u_{k}-u_{i} h_{k}\right)=\frac{v}{P_{M}} \frac{\partial^{2} h_{i}}{\partial x_{k} \partial x_{k}}$,

$\frac{\partial v_{i}}{\partial t}+v_{k} \frac{\partial v_{i}}{\partial x_{k}}=-\frac{k}{m_{s}}\left(v_{i}-u_{i}\right)$

with

$\frac{\partial u_{i}}{\partial x_{i}}=\frac{\partial v_{i}}{\partial x_{i}}=\frac{\partial h_{i}}{\partial x_{i}}=0$

and the equation of energy for an incompressible fluid with constant properties and for negligible frictional heating.

$\frac{\partial T}{\partial t}+u_{i} \frac{\partial T}{\partial x_{i}}=\left(\frac{v}{p_{r}}\right) \frac{\partial^{2} T}{\partial x_{i} \partial x_{i}}$.

The subscripts can take on the values 1, 2 or 3 .

Here,

$\mathrm{u}_{\mathrm{i}}$, turbulent velocity component; hi, magnetic field fluctuation component,

$W(\hat{x}, t)=\frac{p}{\rho}+\frac{1}{2}\left\langle h^{2}\right\rangle+\frac{1}{2}|\hat{\Omega} \times \hat{x}|^{2}, \quad$ total MHD pressure inclusive of potential and centrifugal force,

$p(\hat{x}, t)=$ hydro-dynamic pressure,

$\rho=$ fluid density,

$p_{M}=\frac{v}{\lambda}$, magnetic Prandtl number,

$p_{r}=\frac{v}{\gamma}$, Prandtl number,

$v=$ kinematic viscosity,

$\gamma=\frac{\mathrm{K}}{\rho c_{p}}$, thermal diffusivit $\mathrm{y}$,

$\lambda=(4 \pi \mu \sigma)^{-1}$, magnetic diffusivity,

$\mathrm{c}_{\mathrm{p}}=$ heat capacity at constant pressure ,

$\Omega_{m}=$ constant angular velocity components,

$\epsilon_{m k i}=$ alternating tensor,

$m_{s}=\frac{4}{3} \pi R_{s}^{3} \rho_{s}$, mass of single spherical

dust particle of radius $\mathrm{R}_{\mathrm{s}}$, $\rho_{s}=$ constant density of the material

in dust particle,

$\mathrm{x}_{\mathrm{k}}=$ Space co-ordinate, the subscripts can take on the values 1,2 or 3.

\section{Two-point Correlation and Spectral Equations}

The induction equation of a magnetic field at the point $\mathrm{p}$ is

$$
\frac{\partial h_{i}}{\partial t}+u_{k} \frac{\partial h_{i}}{\partial x_{k}}-h_{k} \frac{\partial u_{i}}{\partial x_{k}}=\frac{v}{p_{M}} \frac{\partial^{2} h_{i}}{\partial x_{k} \partial x_{k}}
$$

and the energy equation at the point $p^{\prime}$ is

$$
\frac{\partial T_{j}^{\prime}}{\partial t}+u_{k}^{\prime} \frac{\partial T_{j}^{\prime}}{\partial x_{k}^{\prime}}=\frac{v}{p_{r}} \frac{\partial^{2} T_{j}^{\prime}}{\partial x_{k}^{\prime} \partial x_{k}^{\prime}} .
$$

The points p and $P^{\prime}$ are separated by the vector $r$ is shown bellow

$$
\mathrm{p} \longrightarrow p^{\prime}
$$

Multiplying equation (6) by $T_{j}^{\prime}$ and (7) by $\mathrm{h}_{\mathrm{i}}$, adding and taking ensemble average, we get

$$
\begin{aligned}
& \frac{\partial\left\langle h_{i} T_{j}^{\prime}\right\rangle}{\partial t}+u_{k} \frac{\partial\left\langle h_{i} T_{j}^{\prime}\right\rangle}{\partial x_{k}}+u_{k}^{\prime} \frac{\partial\left\langle h_{i} T_{j}^{\prime}\right\rangle}{\partial x_{k}^{\prime}}-h_{k} \frac{\partial\left\langle u_{i} T_{j}^{\prime}\right\rangle}{\partial x_{k}^{\prime}} \\
& =v\left[\frac{1}{P_{M}} \frac{\partial^{2}\left\langle h_{i} T_{j}^{\prime}\right\rangle}{\partial x_{k} \partial x_{k}}+\frac{1}{P_{r}} \frac{\partial^{2}\left\langle h_{i} T_{j}^{\prime}\right\rangle}{\partial x_{k}^{\prime} \partial x_{k}^{\prime}}\right] \cdot-\cdot------(8)
\end{aligned}
$$

Angular bracket is used to denote an ensemble average.

The continuity equation is

$\frac{\partial u_{k}}{\partial x_{k}}=\frac{\partial u_{k}^{\prime}}{\partial x_{k}^{\prime}}=0$

Substituting equation (4.4) in to equation (4.3) yields

$$
\begin{aligned}
& \frac{\partial\left\langle h_{i} T_{j}^{\prime}\right\rangle}{\partial t}+\frac{\partial\left\langle u_{k} h_{i} T_{j}^{\prime}\right\rangle}{\partial x_{k}}+\frac{\partial\left\langle u_{k}^{\prime} h_{i} T_{j}^{\prime}\right\rangle}{\partial x_{k}^{\prime}}-\frac{\partial\left\langle u_{i} h_{k} T_{j}^{\prime}\right\rangle}{\partial x_{k}} \\
& =v\left[\frac{1}{p_{M}} \frac{\partial^{2}\left\langle h_{i} T_{j}^{\prime}\right\rangle}{\partial x_{k} d x_{k}}+\frac{1}{p_{r}} \frac{\partial^{2}\left\langle h_{i} T_{j}^{\prime}\right\rangle}{\partial x_{k}^{\prime} \partial x_{k}^{\prime}}\right] \cdot-\cdot----(10)
\end{aligned}
$$

Using the transformations

$\frac{\partial}{\partial r_{k}}=-\frac{\partial}{\partial x_{k}}=\frac{\partial}{\partial x_{k}^{\prime}}$ 
and the Chandrasekhar relations (1951a)

$$
\left\langle u_{k} h_{i} T_{j}^{\prime}\right\rangle=-\left\langle u_{k}^{\prime} h_{i} T_{j}^{\prime}\right\rangle,
$$

in to equation (10) one obtains

$$
\begin{aligned}
& \frac{\partial}{\partial t}\left\langle h_{i} T_{j}^{\prime}\right\rangle+2 \frac{\partial}{\partial r_{k}}\left\langle u_{k}^{\prime} h_{i} T_{j}^{\prime}\right\rangle+\frac{\partial\left\langle u_{i} h_{k} T_{j}^{\prime}\right\rangle}{\partial r_{k}} \\
& =v\left[\frac{\partial^{2}\left\langle h_{i} T_{j}^{\prime}\right\rangle}{\partial r_{k} \partial r_{k}}\left(\frac{1}{P_{M}}+\frac{1}{p_{r}}\right)\right] .
\end{aligned}
$$

Now we write this equation in spectral form in order to reduce it to an ordinary differential equation by use of the following three-dimensional Fourier transforms.

$$
\begin{aligned}
& \left\langle h_{i} T_{j}^{\prime}(\hat{r})\right\rangle=\int_{-\infty}^{\infty}\left\langle\psi_{i} \tau_{j}^{\prime}(\hat{K})\right\rangle \exp [\hat{i}(\hat{K} . \hat{r})] d \hat{K},-(12) \\
& \left\langle u_{i} h_{k} T_{j}^{\prime}(r)\right\rangle=\int_{-\infty}^{\infty}\left\langle\phi_{i} \psi_{k} \tau_{j}^{\prime}(\hat{K})\right\rangle \exp [\hat{i}(\hat{K} \cdot \hat{r})] d \hat{K}, \\
& \left\langle u_{k}^{\prime} h_{i} T_{j}^{\prime}(r)\right\rangle=\left\langle u_{k} h_{i} T_{j}^{\prime}(-\hat{r})\right\rangle \\
& =\int_{-\infty}^{\infty}\left\langle\phi_{k} \psi_{i} \tau_{j}^{\prime}(-\hat{k})\right\rangle \exp [\hat{i}(\hat{k} \cdot \hat{r})] d \hat{K}
\end{aligned}
$$

Equation (14) is obtained by interchanging the subscripts $i$ and $j$ and then the points $\mathrm{p}$ and $p{ }^{\prime}$.

Substituting of equation (12) to (14) in to equation (11) leads to the spectral equation

$$
\begin{aligned}
& \frac{\partial\left\langle\psi_{i} \tau_{j}\right\rangle}{\partial t}+i K_{k}\left[2\left\langle\phi_{k} \psi_{i} \tau_{j}^{\prime}(-\hat{K})+\left\langle\phi_{i} \psi_{k} \tau_{j}^{\prime}(\hat{K})\right\rangle\right]\right. \\
& =-v\left[\left(\frac{1}{p_{M}}+\frac{1}{p_{r}}\right) k^{2}\left\langle\psi_{i} \tau_{j}^{\prime}(\hat{K})\right\rangle\right] .
\end{aligned}
$$

The tensor equation (15) becomes a scalar equation by contraction of the indices $i$ and $j$

$$
\begin{aligned}
& \frac{\partial\left\langle\psi_{i} \tau_{i}^{\prime}(\hat{K})\right\rangle}{\partial t}+i K_{k}\left[2\left\langle\phi_{k} \psi_{i} \tau^{\prime}(-\hat{K})\right\rangle+\left\langle\phi_{i} \psi_{k} \tau_{i}^{\prime}(\hat{K})\right\rangle\right] \\
& =-v\left[\left(\frac{1}{P_{M}}+\frac{1}{P_{r}}\right) k^{2}\left\langle\psi_{i} \tau_{i}^{\prime}(\hat{k})\right\rangle\right]
\end{aligned}
$$

\section{Three-point Correlation and Spectral Equations}

Similar Procedure can be used to find the three-point correlation equation. For this purpose, considering three points $\mathrm{p}, p^{\prime}, p^{\prime \prime}$ separated by the vectors $\hat{r}$ and $\hat{r}^{\prime}$ we take the momentum equation of MHD turbulence at the point $\mathrm{P}$, the induction equation at the point $P^{\prime}$ and the energy equation at $P^{\prime \prime}$ as

$$
\begin{aligned}
& \frac{\partial u_{i}}{\partial t}+u_{k} \frac{\partial u_{i}}{\partial k_{k}}-h_{k} \frac{\partial h_{i}}{\partial x_{k}}=\frac{\partial w}{\partial x_{i}}+\frac{\partial^{2} u_{i}}{\partial x_{k}}-2 \epsilon_{n k i} \Omega_{n i}, \\
& \frac{\partial h_{i}^{\prime}}{\partial t}+u_{k}^{\prime} \frac{\partial h_{i}^{\prime}}{\partial x_{k}}-h_{k}^{\prime} \frac{\partial u_{i}^{\prime}}{\partial x_{k}^{\prime}}=\frac{v}{P_{M}} \frac{\partial^{2} h_{i}^{\prime}}{\partial x_{k}^{\prime} \partial x_{k}^{\prime}}-
\end{aligned}
$$

and

$$
\frac{\partial T_{j}^{\prime \prime}}{\partial t}+u_{k}^{\prime \prime} \frac{\partial T_{j}^{\prime \prime}}{\partial x_{k}^{\prime \prime}}=\left(\frac{v}{P_{r}}\right) \frac{\partial^{2} T_{j}^{\prime \prime}}{\partial x_{k}^{\prime \prime} \partial x_{k}^{\prime \prime}},
$$

where

$W(\hat{x}, t)=\frac{P}{\rho}+\frac{1}{2}\left\langle h^{2}\right\rangle+\frac{1}{2}|\hat{\Omega} \times \hat{x}|^{2}$, total $\quad$ MHD $\quad$ pressure inclusive of potential and centrifugal force $P(\hat{x}, t)$, hydrodynamic pressure; $\Omega m$, constant angular velocity components; $\epsilon_{m k i}$, alternating tensor.

Multiplying equation (17) by $h_{i}^{\prime} T_{j}^{\prime \prime}$, (18) by $u_{i} T_{j}^{\prime \prime}$ and (19) by $u_{i} h_{i}^{\prime}$, adding and taking ensemble average, one obtains

$$
\begin{aligned}
& \frac{\partial\left\langle u_{i} h_{i}^{\prime} T_{j}^{\prime \prime}\right\rangle}{\partial t}+\frac{\partial\left\langle u_{i} u_{k} h_{i}^{\prime} T_{j}\right\rangle}{\partial x_{k}}-\frac{\partial\left\langle h_{i} h_{k} h_{i}^{\prime} T_{j}^{\prime \prime}\right\rangle}{\partial x_{k}^{\prime \prime}} \\
& +\frac{\partial\left\langle u_{i} u_{k}^{\prime} h_{i}^{\prime} T_{j}^{\prime \prime}\right\rangle}{\partial x_{k}^{\prime}}-\frac{\partial\left\langle u_{i} u_{i}^{\prime} h_{k}^{\prime} T_{j}^{\prime \prime}\right\rangle}{\partial x_{k}^{\prime}}+\frac{\partial\left\langle u_{i} h_{i}^{\prime} u_{k}^{\prime \prime} T_{j}^{\prime \prime}\right\rangle}{\partial x_{k}^{\prime \prime}}
\end{aligned}
$$$$
=-\frac{\partial\left\langle w h_{i}^{\prime} T_{j}^{\prime}\right\rangle}{\partial x_{i}}+v \frac{\partial^{2}\left\langle u_{i} h_{i}^{\prime} T_{j}^{\prime \prime}\right\rangle}{\partial x_{k} \partial x_{k}}+
$$$$
\left[\frac{1}{P_{M}} \frac{\partial^{2}\left\langle u_{i} h_{i}^{\prime} T_{j}^{\prime \prime}\right\rangle}{\partial x_{k}^{\prime} \partial x_{k}^{\prime}}+\frac{1}{P_{r}} \frac{\partial^{2}\left\langle u_{i} h_{i}^{\prime} T_{j}^{\prime \prime}\right\rangle}{\partial x_{k}^{\prime \prime} \partial x_{k}^{\prime \prime}}\right]
$$

$$
-2 \epsilon_{m k i} \Omega_{m}\left\langle u_{i} h_{i}^{\prime} T_{j}^{\prime \prime}\right\rangle
$$

Using the transformations

$$
\frac{\partial}{\partial x_{k}}=-\left(\frac{\partial}{\partial r_{k}}+\frac{\partial}{\partial r_{k}^{\prime}}\right) \frac{\partial}{\partial x_{k}^{\prime}}=\frac{\partial}{\partial r_{k}}, \frac{\partial}{\partial x_{k}^{\prime \prime}}=\frac{\partial}{\partial r_{k}^{\prime}}
$$




$$
\begin{aligned}
& \frac{\partial\left\langle u_{i} h_{i}^{\prime} T_{j}^{\prime \prime}\right\rangle}{\partial t}-v\left[\left(1+\frac{1}{p_{M}}\right) \frac{\partial^{2}\left\langle u_{i} h_{i}^{\prime} T_{j}^{\prime \prime}\right\rangle}{\partial r_{k} \partial r_{k}}\right. \\
& \left.+\left(1+\frac{1}{p_{r}}\right) \frac{\partial^{2}\left\langle u_{i} h_{i}^{\prime} T_{j}^{\prime \prime}\right\rangle}{\partial r_{k}^{\prime} \partial r_{k}^{\prime}}+2 \frac{\partial^{2}\left\langle u_{i} h_{i}^{\prime} T_{j}^{\prime \prime}\right\rangle}{\partial r_{k} \partial r_{k}^{\prime}}\right] \\
& =\frac{\partial\left\langle u_{i} u_{k} h_{i}^{\prime} T_{j}^{\prime \prime}\right\rangle}{\partial r_{k}}+\frac{\partial\left\langle u_{i} u_{k} h_{i}^{\prime} T_{j}^{\prime \prime}\right\rangle}{\partial r_{k}^{\prime}}-\frac{\partial\left\langle h_{i} h_{k} h_{i}^{\prime} T_{j}^{\prime \prime}\right\rangle}{\partial r_{k}} \\
& -\frac{\partial\left\langle h_{i} h_{k} h_{i}^{\prime} T_{j}^{\prime \prime}\right\rangle}{\partial r_{k}^{\prime}}-\frac{\partial\left\langle u_{i} u_{k}^{\prime} h_{i}^{\prime} T_{j}^{\prime \prime}\right\rangle}{\partial r_{k}} \\
& +\frac{\partial\left\langle u_{i} u_{i}^{\prime} h_{k}^{\prime} T_{j}^{\prime \prime}\right\rangle}{\partial r_{k}}-\frac{\partial\left\langle u_{i} u_{k}^{\prime \prime} h_{i}^{\prime} T_{j}^{\prime \prime}\right\rangle}{\partial r_{k}^{\prime}}+\frac{\partial\left\langle w h_{i}^{\prime} T_{j}^{\prime \prime}\right\rangle}{\partial r_{i}} \\
& +\frac{\partial\left\langle w h_{i}^{\prime} T_{j}^{\prime \prime}\right\rangle}{\partial r_{i}^{\prime}}-2 \in{ }_{m k i} \Omega_{m}\left\langle u_{i} h_{i}^{\prime} T_{j}^{\prime \prime}\right\rangle \text {---.----- (21) }
\end{aligned}
$$

In order to write the equation (10) to spectral form, we can define the following six dimensional Fourier transforms:

$$
\left\langle u_{i} h_{i}^{\prime}(\hat{r}) T_{j}^{\prime \prime}\left(\hat{r}^{\prime}\right)\right\rangle=\int_{-\infty}^{\infty} \int_{-\infty}^{\infty}\left\langle\phi_{i} \beta_{i}^{\prime}(\hat{k}) \theta_{j}^{\prime \prime}\left(\hat{k}^{\prime}\right)\right\rangle \exp
$$

$\left[\hat{i}\left(\hat{k} \cdot \hat{r}+\hat{k}^{\prime} \cdot \hat{r}^{\prime}\right)\right] d \hat{k} d \hat{k}^{\prime}$

$\left\langle u_{i} u_{k} h_{i}^{\prime}(\hat{r}) T_{j}^{\prime \prime}\left(\hat{r^{\prime}}\right)\right\rangle=\int_{-\infty-\infty}^{\infty} \int_{i}^{\infty}\left\langle\phi_{i} \phi_{k} \beta_{i}^{\prime}(\hat{k}) \theta_{j}^{\prime \prime}\left(\hat{k}^{\prime}\right)\right\rangle \exp$

$\left[\hat{i}\left(\hat{k} \cdot \hat{r}+\hat{k}^{\prime} \cdot \hat{r}^{\prime}\right)\right] d \hat{k} d \hat{k}^{\prime}$

$\left\langle h_{i} h_{k} h_{i}^{\prime}(\hat{r}) T_{j}^{\prime \prime}\left(\hat{r}^{\prime}\right)\right\rangle=\int_{-\infty-\infty}^{\infty} \int_{i}^{\infty}\left\langle\beta_{i} \beta_{k} \beta_{i}^{\prime}(\hat{k}) \theta_{j}^{\prime \prime}\left(\hat{k}^{\prime}\right)\right\rangle \exp$

$\left[\hat{i}\left(\hat{k} \cdot \hat{r}+\hat{k}^{\prime} \cdot \hat{r}^{\prime}\right)\right] d \hat{k} d \hat{k}^{\prime}$

$\left\langle u_{i} u_{k}^{\prime} h_{i}^{\prime}(\hat{r}) T_{j}^{\prime \prime}\left(\hat{r}^{\prime}\right)\right\rangle=\int_{-\infty-\infty}^{\infty} \int_{i}^{\infty}\left\langle\phi_{i} \phi_{k}^{\prime}(\hat{k}) \beta_{i}^{\prime}(\hat{k}) \theta_{j}^{\prime \prime}\left(\hat{k}^{\prime}\right)\right\rangle \exp$

$\left[\hat{i}\left(\hat{k} \cdot \hat{r}+\hat{k}^{\prime} \cdot \hat{r}^{\prime}\right)\right] d \hat{k} d \hat{k}^{\prime}$

$$
\begin{aligned}
& \left\langle u_{i} u_{i}^{\prime}(\hat{r}) h_{k}^{\prime}(\hat{r}) T_{j}^{\prime \prime}\left(\hat{r^{\prime}}\right)\right\rangle=\int_{-\infty}^{\infty} \int_{-\infty}^{\infty}\left\langle\phi_{i} \phi_{i}^{\prime} \beta_{k}^{\prime}(\hat{k}) \theta_{j}^{\prime \prime}\left(\hat{k^{\prime}}\right)\right\rangle \exp \\
& {\left[\hat{i}\left(\hat{k} \cdot \hat{r}+\hat{k^{\prime} \cdot \hat{r}^{\prime}}\right)\right] d \hat{k} d \hat{k}^{\prime}} \\
& \left\langle w h_{i}^{\prime}(\hat{r}) T_{j}^{\prime \prime}\left(\hat{r^{\prime}}\right)\right\rangle=\int_{-\infty-\infty}^{\infty} \int_{-\infty}^{\infty}\left\langle\gamma \beta_{i}^{\prime}(\hat{k}) \theta_{j}^{\prime \prime}\left(\hat{k^{\prime}}\right)\right\rangle \exp \\
& {\left[\hat{i}\left(\hat{k} \cdot \hat{r}+\hat{k}^{\prime} \cdot \hat{r}^{\prime}\right)\right] d \hat{k} d \hat{k}^{\prime}}
\end{aligned}
$$

Interchanging the points $p^{\prime}$ and $p^{\prime \prime}$ along with the subscripts $i$ and $j$,

$$
\left\langle u_{i} u_{k}^{\prime \prime} h_{i}^{\prime} T_{j}^{\prime \prime}\right\rangle=\left\langle u_{i} u_{k}^{\prime} h_{i}^{\prime} T_{j}^{\prime \prime}\right\rangle \text {. }
$$

By use this fact we can write equation (21) in the form

$$
\begin{aligned}
& \frac{\partial\left\langle\phi_{i} \beta_{i}^{\prime} \theta_{j}^{\prime \prime}\right\rangle}{\partial t}+v\left[\left(1+\frac{1}{p_{M}}\right) k^{2}+\left(1+\frac{1}{p_{r}}\right) k^{2}+2 k_{k} k_{k}^{\prime}-\right. \\
& \left.+\frac{2 \epsilon_{m k i} \Omega_{m}}{v}\right]\left\langle\phi_{i} \beta_{i}^{\prime} \theta_{j}^{\prime \prime}\right\rangle \\
& =i\left(k_{k}+k_{k}^{\prime}\right)\left\langle\phi_{i} \phi_{k} \beta_{i}^{\prime} \theta_{j}^{\prime \prime}\right\rangle-i\left(k_{k}+k_{k}^{\prime}\right)\left\langle\beta_{i} \beta_{k} \beta_{i}^{\prime} \theta_{j}^{\prime \prime}\right\rangle \\
& -i\left(k_{k}+k_{k}^{\prime}\right)\left\langle\phi_{i} \phi_{k}^{\prime} \beta_{i}^{\prime} \theta_{j}^{\prime \prime}\right\rangle \\
& +i k_{k}\left\langle\phi_{i} \phi_{i}^{\prime} \beta_{k}^{\prime} \theta_{j}^{\prime \prime}\right\rangle+i\left(k_{i}+k_{i}^{\prime}\right)\left\langle\gamma \beta_{i}^{\prime} \theta_{j}^{\prime \prime}\right\rangle .
\end{aligned}
$$

The tensor equation (28) can be converted to scalar equation by contraction of the indices $i$ and $j$

$$
\begin{aligned}
& \frac{\left\langle\partial\left\langle\phi_{i} \beta_{i}^{\prime} \theta_{i}^{\prime}\right\rangle\right.}{\partial t}+v\left[\left(1+\frac{1}{p_{M}}\right) k^{2}+\left(1+\frac{1}{p_{r}}\right) k^{2} .\right. \\
& \left.+2 k_{k} k_{k}^{\prime}+2 \frac{\in_{m k i} \Omega_{m}}{v}\right]\left\langle\phi_{i} \beta_{i}^{\prime} \theta_{i}^{\prime \prime}\right\rangle \\
& =i\left(k_{k}+k_{k}^{\prime}\right)\left\langle\phi_{i} \phi_{k} \beta_{i}^{\prime} \theta_{i}^{\prime \prime}\right\rangle-i\left(k_{k}+k_{k}^{\prime}\right) \\
& \left\langle\beta_{i} \beta_{k} \beta_{i}^{\prime} \theta_{i}^{\prime}\right\rangle-i\left(k_{k}+k_{k}^{\prime}\right)\left\langle\phi_{i} \phi_{k} \beta_{i}^{\prime} \theta_{i}^{\prime}\right\rangle \\
& +i k_{k}\left\langle\phi_{i} \phi_{i}^{\prime} \beta_{k}^{\prime} \theta_{i}^{\prime}\right\rangle+i\left(k_{i}+k_{i}^{\prime}\right)\left\langle\gamma \beta_{i}^{\prime} \theta_{i}^{\prime}\right\rangle .
\end{aligned}
$$

If the derivative with respect to $\mathrm{x}_{\mathrm{i}}$ is taken of the momentum equation (17) for the point $p$, the equation multiplied through 
by $\mathrm{h}_{\mathrm{i}} T_{j}$ " and taken time average, the resulting equation

$$
\begin{gathered}
-\frac{\partial^{2}\left\langle w h_{i}^{\prime} T_{j}^{\prime \prime}\right\rangle}{\partial x_{i} \partial x_{i}}=\frac{\partial^{2}}{\partial x_{i} \partial x_{k}} \\
\left(\left\langle u_{i} u_{k} h_{i}^{\prime} T_{j}^{\prime \prime}\right\rangle-\left\langle h_{i} h_{k} h_{i}^{\prime} T_{j}^{\prime \prime}\right\rangle\right)
\end{gathered}
$$

Writing this equation in terms of the independent variables $\hat{r}$ and $\hat{r}^{\prime}$

$$
\begin{aligned}
& -\left[\frac{\partial^{2}}{\partial r_{i} \partial r_{i}}+2 \frac{\partial^{2}}{\partial r_{i} \partial r_{i}^{\prime}}+\frac{\partial^{2}}{\partial r_{i}^{\prime} \partial r_{i}^{\prime}}\right]\left\langle w h_{i}^{\prime} T_{j}^{\prime}\right\rangle \\
& =\left[\frac{\partial^{2}}{\partial r_{i} \partial r_{k}}+\frac{\partial^{2}}{\partial r_{i} \partial r_{k}}+\frac{\partial^{2}}{\partial r_{i} \partial r_{k}^{\prime}}+\frac{\partial^{2}}{\partial r_{i}^{\prime} \partial r_{k}^{\prime}}\right] \\
& \times\left(\left\langle u_{i} u_{k} h_{i}^{\prime} T_{j}^{\prime}\right\rangle-\left\langle h_{i} h_{k} h_{i}^{\prime} T_{j}^{\prime}\right\rangle\right) .
\end{aligned}
$$

Now taking the Fourier transforms of equation (31), we get $-\left\langle\gamma \beta_{i}^{\prime} \theta_{j}^{\prime \prime}\right\rangle=$

$\frac{\left(k_{i} k_{k}+k_{i}^{\prime} k_{k}+k_{i} k_{k}^{\prime}+k_{i}^{\prime} k_{k}^{\prime}\right)\left(\left\langle\phi_{i} \phi_{k} \beta_{i}^{\prime} \theta_{j}^{\prime \prime}\right\rangle-\left\langle\beta_{i} \beta_{k} \beta_{i}^{\prime} \theta_{j}^{\prime \prime}\right\rangle\right)}{k_{i} k_{i}+2 k_{i}^{\prime} k_{i}+k_{i}^{\prime} k_{i}^{\prime}}$.

Equation (32) can be used t o eliminate $\left\langle\gamma \beta_{i}^{\prime} \theta_{j}^{\prime \prime}\right\rangle$ from equation (28).

\section{Solution for times before the final period}

It is known that equation for final period of decay is obtained by considering the two-point correlations after neglecting the 3rd order correlation terms. To study the decay for times before the final period, the three point correlations are considered and the quadruple correlation terms are neglected because the quadruple correlation terms decays faster than the lower-order correlation terms. Equation (32) shows that term $\left\langle\gamma \beta_{i}^{\prime} \theta_{j}^{\prime \prime}\right\rangle$ associated with the pressure fluctuations

should also be neglected. Thus neglecting all the terms on the right hand side of equation (29)

$$
\begin{gathered}
\frac{\partial\left\langle\phi_{i} \beta_{i}^{\prime} \theta_{i}^{\prime \prime}\right\rangle}{\partial t}+v\left[\left(1+\frac{1}{p_{M}}\right) k^{2}+\left(1+\frac{1}{p_{r}}\right) k^{2}+2 k_{k} k_{k}^{\prime} .\right. \\
\left.+\frac{2 \epsilon_{m k i} \Omega_{m}}{v}\right]\left\langle\phi_{i} \beta_{i}^{\prime} \theta_{i}^{\prime \prime}\right\rangle=0
\end{gathered}
$$

Integrating the equation (33) between $t_{0}$ and $t$ with inner multiplication by $\mathrm{k}_{\mathrm{k}}$ and gives

$$
\begin{aligned}
& k_{k}\left\langle\phi_{i} \beta_{i}^{\prime} \theta_{i}^{\prime \prime}\right\rangle=k_{k}\left[\phi_{i} \beta_{i}^{\prime} \theta_{i}^{\prime \prime}\right]_{0} \exp \left[-v\left\{\left(1+\frac{1}{p_{M}}\right) k^{2}+\right.\right. \\
& \left.\left.\left(1+\frac{1}{p_{r}}\right) k^{\prime 2}+2 k k^{\prime} \cos \theta+\frac{2 \epsilon_{m k i} \Omega_{m}}{v}\right\}\left(t-t_{0}\right)\right]
\end{aligned}
$$

where $\theta$ is the angle between $k$ and $k^{\prime}$ and $\left\langle\phi_{i} \beta_{i}^{\prime} \theta_{i}^{\prime \prime}\right\rangle_{0}$ is the value of $\left\langle\phi_{i} \beta_{i}^{\prime} \theta_{i}^{\prime \prime}\right\rangle$ at $\mathrm{t}=\mathrm{t}_{\mathrm{o}}$.

Now by letting $r^{\prime}=0$ in equation (22) and comparing with equations (13) and (14), we get

$$
\begin{aligned}
& \left\langle\phi_{i} \psi_{k} \tau_{i}^{\prime}(\hat{k})\right\rangle=\int_{-\infty}^{\infty}\left\langle\phi_{i} \beta_{i}^{\prime} \theta_{i}^{\prime \prime}\right\rangle d \hat{k^{\prime}}, \\
& \left\langle\phi_{i} \psi_{i} \tau_{i}^{\prime}(-\hat{k})\right\rangle=\int_{-\infty}^{\infty} \phi_{k} \beta_{i}^{\prime}(-\hat{k}) \theta_{i}^{\prime \prime}\left(-\hat{k}^{\prime}\right) d \hat{k^{\prime}} .
\end{aligned}
$$

Substituting equation (34) to (36) in equation (16)

$$
\begin{aligned}
& \frac{\partial\left\langle\psi_{i} \tau_{i}^{\prime}(\hat{k})\right\rangle}{\partial t}+v\left(\frac{1}{p_{M}}+\frac{1}{p_{r}}\right) k^{2}\left\langle\psi_{i} \tau_{i}^{\prime}(\hat{k})\right\rangle= \\
& \left.-\int_{-\infty}^{\infty} i k_{k}\left[\phi_{i} \beta_{i}^{\prime} \theta_{i}^{\prime \prime}\right\rangle+2\left\langle\phi_{k} \beta_{i}^{\prime}(-\hat{k}) \theta_{i}^{\prime \prime}\left(-\hat{k}^{\prime}\right)\right\rangle\right]_{0} \\
& \quad \exp \left[-v\left(t-t_{o}\right)\left\{\left(1+\frac{1}{p_{M}}\right) k^{2}+\left(1+\frac{1}{p_{r}}\right) k^{\prime 2}\right.\right. \\
& \left.\left.+2 k k^{\prime} \cos \theta+2 \frac{\in_{m k i} \Omega_{m}}{v}\right\}\right] d \hat{k}^{\prime}
\end{aligned}
$$

Now, $d \hat{k}^{\prime}$ can be expressed in terms of $k^{\prime}$ and $\theta$ as $-2 \pi k^{2} d(\cos \theta) d k^{\prime}$ (cf. Deissler ${ }^{1}$ )

Henc $d \hat{k^{\prime}}=-2 \pi k^{\prime 2} d(\cos \theta) d \hat{k^{\prime}}$.

Putting equation (38) in equation (37) yields

$$
\begin{aligned}
& \frac{\partial\left\langle\psi_{i} \tau_{i}^{\prime}(\hat{k})\right\rangle}{\partial t}+v\left(\frac{1}{p_{M}}+\frac{1}{p_{r}}\right) k^{2}\left\langle\psi_{i} \tau_{i}^{\prime}(\hat{k})\right\rangle= \\
& \left.-\int_{0}^{\infty} 2 \pi i k_{k}\left[\phi_{i} \beta_{i}^{\prime} \theta_{i}^{\prime \prime}\right\rangle+2\left\langle\phi_{k} \beta_{i}^{\prime}(-\hat{k}) \theta_{i}^{\prime \prime}\left(-\hat{k^{\prime}}\right)\right\rangle\right]_{0} k^{\prime 2} \times
\end{aligned}
$$


$\left[\int_{-1}^{1} \exp \left\{-v\left(t-t_{o}\right)\left(\left(1+\frac{1}{p_{M}}\right) k^{2}+\left(1+\frac{1}{p_{r}}\right) k^{2}+\right.\right.\right.$

$\left.\left.2 k k^{\prime} \cos \theta+2 \frac{\in_{m k i} \Omega_{m}}{v}\right]\right\} d(\cos \theta) \hat{d k^{\prime}}$

In order to find the solution completely and following Loeffler and Deissler (1961) we assume that

$i k_{k}\left[\left\langle\phi_{i} \beta_{i}^{\prime} \theta_{i}^{\prime}\right\rangle+2\left\langle\phi_{k} \beta_{i}^{\prime}(-\hat{k}) \theta_{i}^{\prime \prime}\left(-\hat{k^{\prime}}\right)\right\rangle\right]_{0}=$ $\frac{\beta_{0}}{(2 \pi)^{2}}\left(k^{2} k^{\prime 4}-k^{4} k^{\prime 2}\right)$

where $\beta_{0}$ is a constant depending on the initial conditions. Substituting equation (40) into equation (39) and completing the integration with respect to $\cos \theta$, one obtains

$\frac{\partial\left(2 \pi\left\langle\psi_{i} \tau_{i}^{\prime}(\hat{k})\right\rangle\right)}{\partial t}+v\left(\frac{1}{p_{M}}+\frac{1}{p_{r}}\right) k^{2}\left(2 \pi\left\langle\psi_{i} \tau_{i}^{\prime}(\hat{k})\right\rangle\right)=$

$-\frac{\beta_{0}}{2 v\left(t-t_{o}\right)} \int_{0}^{\infty}\left(k^{3} k^{\prime 5}-k^{5} k^{3}\right) \times$

$\left[\exp \left\{-v\left(t-t_{o}\right)\left[\left(1+\frac{1}{p_{M}}\right) k^{2}+\left(1+\frac{1}{p_{r}}\right) k^{\prime 2}-2 k k^{\prime}+\right.\right.\right.$

$\left.\left.\frac{2 \in_{m k i} \Omega_{m}}{v}\right]\right\}-\exp \left\{-v\left(t-t_{o}\right)\left[\left(1+\frac{1}{p_{M}}\right) k^{2}+\left(1+\frac{1}{p_{r}}\right) k^{\prime 2}\right.\right.$

$\left.\left.+2 k k^{\prime}+2 \frac{\in_{m k i} \Omega_{m}}{v}\right]\right\} d k^{\prime}$

Multiplying both sides of equation (41) by $k^{2}$, we get

$\frac{\partial Q}{\partial t}+v\left(\frac{1}{p_{M}}+\frac{1}{p_{r}}\right) k^{2} Q=F$,

where, $\mathrm{Q}=2 \pi k^{2}\left\langle\psi_{i} \tau_{i}^{\prime}(\hat{k})\right\rangle$,

$\mathrm{Q}$ is the Magnetic energy Spectrum function.

and

$F=-\frac{\beta_{0}}{2 v\left(t-t_{0}\right)} \int_{0}^{\infty}\left(k^{3} k^{5}-k^{5} k^{3}\right) \times$

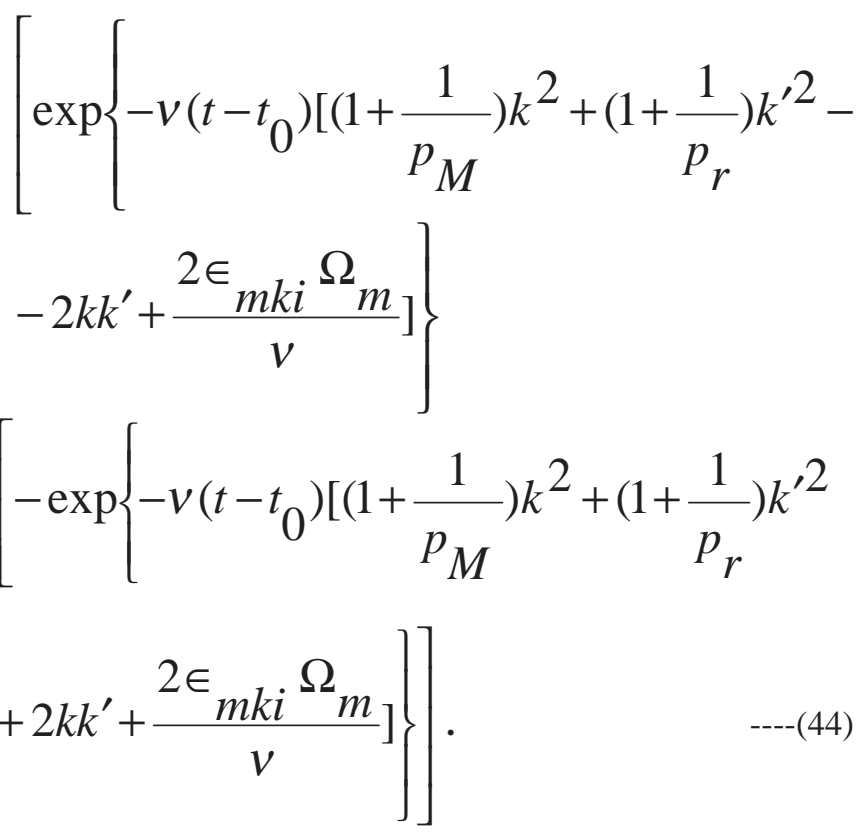

Integrating equation (44) with respect to $k^{\prime}$, we have

$$
\begin{aligned}
& F=-\frac{\beta_{0} \sqrt{\pi} P_{r}^{5 / 2}}{2 v^{3 / 2}\left(t-t_{o}\right)\left(1+p_{r}\right)^{5 / 2}} \exp \left[-2 \frac{\in_{m k i} \Omega_{m}}{v}\left(t-t_{o}\right)\right] \times \\
& \exp \left[-v\left(t-t_{o}\right)\left(1+\frac{1}{p_{M}}-\frac{p_{r}}{1+p_{r}}\right) k^{2}\right] \\
& {\left[\frac{15 p_{r} k^{4}}{4 v^{2}\left(t-t_{o}\right)^{2}\left(1+p_{r}\right)}+\left\{\frac{5 p_{r}{ }^{2}}{\left(1+p_{r}\right)^{2}}-\frac{3}{2}\right\} \frac{k^{6}}{v\left(t-t_{o}\right)} .\right.} \\
& \left.+\left\{\frac{p_{r}{ }^{3}}{\left(1+p_{r}\right)^{3}}-\frac{p_{r}}{\left(1+p_{r}\right)}\right\} k^{8}\right]
\end{aligned}
$$

The series of equation (45) contains only even powers of $k$ and start with $k^{4}$ and the equation represents the transfer function arising owing to consideration of magnetic field at three points at a time.

It is interesting to note that if we integrate equation (44) over all wave numbers, we find that

$\int_{0}^{\infty} F d k=0$

which is indicating that the expression for F satisfies the condition of continuity and homogeneity.

The linear equation (42) can be solved to give 
$Q=\exp \left[-v k^{2}\left(\frac{1}{p_{M}}+\frac{1}{p_{r}}\right)\left(t-t_{O}\right)\right] \int F$

$\exp \left[v k^{2}\left(\frac{1}{p_{M}}+\frac{1}{p_{r}}\right)\left(t-t_{O}\right)\right] d t$

$+J(k) \exp \left[-v k^{2}\left(\frac{1}{p_{M}}+\frac{1}{p_{r}}\right)\left(t-t_{O}\right)\right]$,

where $\mathrm{J}(\mathrm{K})=\frac{N_{0} k^{2}}{\pi}$ is a constant of integration. Substituting the values of $\mathrm{F}$ from equation (45) in to equation (47) and integrating with respect to t, we get

$Q(\hat{k, t})=\frac{N_{0} k^{2}}{\pi} \exp \left[-v k^{2}\left(\frac{1}{p_{M}}+\frac{1}{p_{r}}\right)\left(t-t_{o}\right)\right]$

$+\frac{\beta_{0} \sqrt{\pi} p_{r}^{3 / 2}}{2 v^{3 / 2}\left(1+p_{r}\right)^{7 / 2}} \times \exp \left[-\left\{2 \epsilon_{m k i} \Omega_{m}\left(t-t_{o}\right)\right\}\right]$

$\exp \left[-v k^{2}\left(t-t_{o}\right)\left\{\frac{1+p_{r}+p_{M}}{p_{M}\left(1+p_{r}\right)}\right\}\right]$

$\left[\frac{3 p_{r} k^{4}}{2 v^{2}\left(t-t_{o}\right)^{5 / 2}}+\frac{p_{r}\left(7 p_{r}-6\right) k^{6}}{3 v\left(1+p_{r}\right)\left(t-t_{o}\right)^{3 / 2}}\right.$

$\left.-\frac{4\left(3 p_{r}{ }^{2}-2 p_{r}+3\right) k^{8}}{3\left(1+p_{r}\right)^{2}\left(t-t_{o}\right)^{1 / 2}}+\frac{8 \sqrt{v}\left(3 p_{r}{ }^{2}-2 p_{r}+3\right) k^{9}}{3\left(1+p_{r}\right)^{5 / 2} \sqrt{p_{r}}} N(\omega)\right]$

where $N(\omega)=e^{-\omega^{2}} \int_{0}^{\omega} e^{x^{2}} d x$

and $\omega=k \sqrt{\frac{\lambda\left(t-t_{o}\right)}{p_{r}\left(1+p_{r}\right)}}$.

The function $\mathrm{N}_{(\omega)}$ has been calculated numerically and tabulated in Sarker and Islam (2001).

By setting $\hat{r}=0, j=i, d \hat{K}=-2 \pi k^{2} d(\cos \theta) d k$ and $Q=2 \pi k^{2}\left\langle\psi_{i} \psi_{i}^{\prime}(\hat{K})\right\rangle$ in equation (12), we get the expression for temperature energy decay as

$\frac{\left\langle T^{2}\right\rangle}{2}=\frac{T_{i} T_{i}^{\prime}}{2}=\int_{0}^{\infty} Q(\hat{k}) d \hat{k}$
Substituting equations (48) in to (49) and after integration, we get

$$
\begin{aligned}
& \frac{\left\langle T^{2}\right\rangle}{2}=\frac{N_{o} p_{r}{ }^{3 / 2} p_{M}{ }^{3 / 2}\left(t-t_{o}\right)^{-3 / 2}}{4 \sqrt{\pi} v^{3 / 2}\left(p_{r}+p_{M}\right)^{3 / 2}}+\exp \left[-2 \epsilon_{m k i} \Omega_{m}\right] \\
& \times \frac{\beta_{0} \pi p_{r}{ }^{7 / 2} p_{M}{ }^{5 / 2}\left(t-t_{o}\right)^{-5}}{2 v^{6}\left(1+p_{r}\right)\left(1+p_{r}+p_{M}\right)^{5 / 2}} \times \\
& \left\{\frac{9}{16}+\frac{5 p_{M}\left(7 p_{r}-6\right)}{16\left(1+p_{r}+p_{M}\right)}-\frac{35 p_{M}^{2}\left(3 p_{M}^{2}-2 p_{r}+3\right)}{8 p_{r}\left(1+p_{r}+p_{M}\right)^{2}}+\right.
\end{aligned}
$$

$\left.\cdot \frac{8 p_{M}^{3}\left(3 p_{r}^{2}-2 p_{r}+3\right)}{3.2^{6} \cdot p_{r}^{2}\left(1+p_{r}+p_{M}\right)^{3}} \sum_{n=0}^{\infty} \frac{1.3 .5 \ldots . .(2 n+9)}{n !(2 n+1) 2^{2 n}\left(1+p_{r}\right)^{n}}\right\}$

or

$$
\begin{aligned}
& \frac{\left\langle T^{2}\right\rangle}{2}=\frac{N_{0} P_{r}^{3 / 2} p_{M}^{3 / 2}\left(t-t_{o}\right)^{-3 / 2}}{4 \sqrt{\pi} v^{3 / 2}\left(p_{r}+p_{M}\right)^{3 / 2}}+ \\
& \beta_{0} z v^{-6}\left(t-t_{o}\right)^{-5} \times \exp \left[-2 \epsilon_{m k i} \Omega_{m}\right],
\end{aligned}
$$

where

$$
\begin{aligned}
& Z=\frac{\pi p_{r}^{7 / 2} p_{M}^{5 / 2}}{2\left(1+p_{r}\right)\left(1+p_{r}+p_{M}\right)^{5 / 2}} \times \\
& {\left[\frac{9}{16}+\frac{5 P_{M}\left(7 P_{r}-6\right)}{16\left(1+P_{r}+p_{M}\right)}-\frac{35 p_{M}^{2}\left(3 p_{r}^{2}-2 p_{r}+3\right)}{8 p_{r}\left(1+p_{r}+p_{M}\right)^{2}}+\right.} \\
& \left.\frac{8 p_{M}^{3}\left(3 p_{r}^{2}-2 p_{r}+3\right)}{3.2^{6} p_{r}^{2}\left(1+p_{r}+p_{M}\right)^{3}} \sum_{n=0}^{\infty} \frac{1.3 .5 \ldots(2 n+9)}{n !(2 n+1) 2^{2 n}\left(1+p_{r}\right)^{n}}\right] .
\end{aligned}
$$

Thus the energy decay law for temperature field fluctuations of MHD turbulence in a rotating system before the final period may be written as

$$
\begin{aligned}
& \left\langle T^{2}\right\rangle=X\left(t-t_{o}\right)^{-3 / 2}+\exp \\
& {\left[-\left\{2 \in_{m k i} \Omega_{m}\right\}\right] \mathrm{Y}\left(t-t_{o}\right)^{-5},}
\end{aligned}
$$

where

$$
X=\frac{N_{0} P_{r}^{3 / 2} p_{M}^{3 / 2}}{2 \sqrt{\pi} v^{3 / 2}\left(p_{r}+p_{M}\right)^{3 / 2}} \text { and } \mathrm{Y}=2 \beta_{0} Z v^{-6}
$$

$\left\langle\mathrm{T}^{2}\right\rangle$ is the total "energy" (the mean square of the temperature fluctuations) $t$ is the time, $x$ and $t_{0}$ are constants determined by the initial conditions. The constant $\mathrm{Y}$ depends on both initial conditions and the fluid Prandtl number. 


\section{Results and Discussion}

In equation (51) we obtained the decay law of temperature fluctuations in MHD turbulence before the final period in a rotating system considering three-point correlation equation after neglecting quadruple correlation terms. If the system is non-rotating, then $\Omega_{\mathrm{m}}=0$ the equation (50) becomes.

$$
\left\langle T^{2}\right\rangle=X\left(t-t_{o}\right)^{-3 / 2}+\mathrm{Y}\left(t-t_{o}\right)^{-5}
$$

which was obtained earlier by Sarker and Rahman (1998)

In the absence of a magnetic field, magnetic Prandtl number coincides with the Prandtl number (i.e. $\mathrm{P}_{\mathrm{r}}=\mathrm{PM}$ ) and the system is non rotating the equation (50) becomes

$$
\frac{\left\langle T^{2}\right\rangle}{2}=\frac{N_{0} p_{r}^{3 / 2}}{8 \sqrt{2 \pi} v^{3 / 2}\left(t-t_{o}\right)^{3 / 2}}+\frac{\beta_{0} Z}{v^{6}\left(t-t_{o}\right)^{5}}
$$

which was obtained earlier by Loeffler and Deissler (1961).

We conclude that due to the effect of rotation of fluid in the flow field, the turbulent energy decays more rapidly than the energy for non-rotating fluid. The 1st term of the right hand side of equation (51) corresponds to the temperature energy for two-point correlation and second term represents temperature energy for three-point correlation. For large times the last term in the equation (51) becomes negligible, leaving the $-3 / 2$ power decay law for the final period. If we considering the higher order correlation terms in the analysis, it appears that more terms in higher power of time would be added to the equation (51).

\section{References}

Azad M. A. K., Sarker, M. S. A. and Mondol M. N. I. (2006) Decay of temperature fluctuations in dusty fluid MHD turbulence before the final period in a rotating system, Journal of Engineering and Applied Sciences (JEAS), Medwell online, Pakistan, 1(2): 187-194 .

Azad M. A. K. and Sarker M. S. A. (2003) Decay of MHD turbulence before the final period for the case of multipoint and multi-time in presence of dust particle, BJSIR, 38(3-4): 151-164.

Chandrasekhar S. (1951a) The invariant theory of isotropic turbulence in magneto-hydrodynamics, Proc. Roy. Soc., London, A204: 435-449.

Deissler R. G. (1958) On the decay of homogeneous turbulence before the final period, Phys. Fluid, 1: 111-121 .

Deissler R. G. (1960) A theory of decaying homogeneous turbulence, Phys. Fluid, 3: 176-187.
Loeffler A. L. and Deissler R. G. (1961) Decay of temperature fluctuations in homogeneous turbulence before the final period, Int. J. Heat Mass Transfer, 1: 312-324 .

Kumar P. and Patel. S. R. (1975) One first-order reactant in homogeneous turbulence, Int. J. Engeng. Sci., 13: 305-315.

Sarker M. S. A. and Islam M. A. (2001) Decay of MHD turbulence before the final period for the case of multipoint and multi-time, Indian J. Pure Appl. Math., 32(7): 1065-1076.

Islam M. A. and Sarker M. S. A. (2001) First-order reactant in MHD turbulence before the final period of decay for the case of multi-point and multi-time, Indian J. Pure Appl. Math., 32(8): 1173-1184.

Sarker M. S. A. and Rahman M. L. (1998) Decay of temperature fluctuations in MHD turbulence before the final period, North Bengal Univ. Review (Sci. and Tech.), India, 9: 91-102.

Sarker M. S. A. and Islam M. A. (2001) Decay of temperature fluctuations in homogeneous turbulence before the final period for the case of multi-point and multi-time, Ph. D. Thesis, Dept. of Mathematics, R. U., 27-40.

Sarker M. S. A. and Kishore, N. (1991) Decay of MHD turbulence before the final period, Int. J. Engeng. Sci., 29: 1479-1485.

Kishore N. and Dixit T. (1979) The effect of coriolis force on acceleration covariance in isotropic hydro-magnetic turbulence, Scientific Research, B.H.U., 30(2): 305-314.

Kishore N. and Singh S. R. (1984) The effect of coriolis force on acceleration covariance in turbulent flow with rotational symmetry, Astrophysics and Space Sci., 104: 121-125 .

Sarker M. S. A. and Islam M. A. (2001) Decay of dusty fluid turbulence before the final period in a rotating system, J. Math. and Math. Sci., 16: 35-48.

Shimomura Y. and Yoshizawa A. (1986) Statistical analysis of an isotropic turbulent viscosity in a rotating system, J. Physical Soc. Japan, 55: 1904-1917 .

Shimomura Y. (1986) Two-scale direct-interaction approach to the turbulent scalar flux in a rotating system, $J$. Physical Soc. Japan, 55: 3388-3401.

Shimomura Y. (1989) A statistical derived two-equation model of turbulent scalar flux in a rotating system, $J$. Physical Soc. Japan, 58: 352-355.

Received : July 29, 2008;

Accepted : January 15, 2009 